\title{
COUPLED PAIRS OF DUAL INTEGRAL EQUATIONS WITH TRIGONOMETRIC KERNELS*
}

\author{
BY \\ R. KHADEM** (Université Laval, Quebec) \\ AND \\ L. M. KEER (Northwestern University, Evanston, Illinois)
}

\begin{abstract}
The solution is given to a system of two pairs of dual integral equations with constant coefficients involving trigonometric kernels. The method is analogous to that applied to Bessel function kernels and involves reduction to a single WienerHopf equation for which a solution is available. The example of an indenter moving with friction present is worked out by this method and also by means of equivalent reduction of the system of equations to a singular integral equation.
\end{abstract}

1. Introduction. This paper deals with the following system of coupled pairs of dual integral equations with trigonometric kernels:

$$
\begin{array}{ll}
\int_{0}^{\infty} t^{-1}\left[a_{1} f(t)+a_{2} g(t)\right] \cos x t d t=h_{1}(x), & x<1, \\
\int_{0}^{\infty}\left[a_{3} f(t)+a_{4} g(t)\right] \cos x t d t=h_{2}(x), & x>1, \\
\int_{0}^{\infty} t^{-1}\left[b_{1} f(t)+b_{2} g(t)\right] \sin x t d t=s_{1}(x), & x<1, \\
\int_{0}^{\infty}\left[b_{3} f(t)+b_{4} g(t)\right] \sin x t d t=s_{2}(x), & x>1,
\end{array}
$$

where $h_{i}(x), s_{i}(x)(i=1,2)$ are known functions and $a_{i}, b_{i}(i=1,2,3,4)$ are known coefficients. If $a_{i}$ and $b_{i}(i=1,3)$ are assumed to be non-zero or if the system defined by (1) can be so transformed that the latter condition is satisfied, then the above system is analogous to one with Bessel function kernels [1], and the same method applies. The number of constants in (1.1) can be reduced without loss of generality by dividing the equations respectively by $a_{1}, a_{3}, b_{1}$ and $b_{3}$ or $a_{2}, a_{4}, b_{2}$ and $b_{4}$, and redefining the functions $h_{i}(x)$ and $s_{i}(x)$ accordingly. However, inasmuch as some of the above constants may be zero, and in the interest of comparing the analysis of the present work with [1], the authors prefer to maintain the constants as in (1.1).

Solutions $f(t)$ and $g(t)$ are sought belonging to the class $L^{1}(-\infty, \infty)$. To this end the system (1.1) is reduced to the single integral equation of the Wiener-Hopf type

\footnotetext{
* Received July 3, 1972; revised version received November 16, 1972. This work has been supported by the National Research Council of Canada Grant A 7847.

** Present address: School of Planning and Computer Applications, Teheran, Iran.
} 


$$
g(t)=c(t)+\beta_{1} \int_{0}^{\infty} g(u) k(u+t) d u+\beta_{2} \int_{0}^{\infty} g(u) k(u-t) d u, \quad t>0,
$$

where $k(t)=\sin t / \pi t, c(t)$ is a known function expressed in terms of $h_{i}(x)$ and $s_{i}(x)$ $(i=1,2)$ in $(1.1)$ and $\beta_{1}, \beta_{2}$ are known constants.

The system (1.1) is suitably transformed so that $\beta_{1}=0$, for which case Spence [2] gives a solution for $c(t)$ of a particular form and for real coefficients; in [1] the solution has been modified for complex coefficients. Hence, by using the known solution of (1.2) with $\beta_{1}=0$, the system of coupled pairs of equations (1.1) is resolved.

Application. The solution obtained in this paper is applicable to a number of problems arising from elasticity. Recently, there have been several solutions to problems involving the steady motion of indenters developed by means of the complex variable technique by Craggs and Roberts [3] and Roberts [4], [5]. The solution techniques presented herein give a slightly different approach which may be more useful when more difficult problems, such as those involving layered media, are considered. The authors note that others have solved related problems by use of Fourier transforms to reduce the physical problem to a singular integral equation. Brilla [6] has solved adhesion problems for anisotropic halfplanes and Alblas and Kuipers [7], [8], [9], and [10] have solved several problems for the identation of elastic layers. Keer and Freedman [11] have used a method similar to that presented here to determine static compliances for a rigid strip bonded to an elastic layer.

The particular case of a rough identor in steady motion with velocity $c(c<$ velocity of Rayleigh waves) on an elastic halfspace is solved in detail. The boundary-value problem may be formulated in terms of a pair of simultaneous dual integral equations of the form given by (1) with $a_{1}=b_{2}=d, a_{2}=-b_{1}=p, a_{4}=b_{3}=0$ and $h_{2}(x)=$ $s_{2}(x)=0$, where $h_{1}(x)$ and $s_{1}(x)$ are appropriate for a cylindrical and a wedge punch, which are the two specific geometries studied. By use of (1.2) the problem is solved and appropriate physical quantities calculated. It is shown that in the special case $c=0$ the wedge solution reduces to that given by Mushkhelishvili [13].

By writing $f(t)$ and $g(t)$ in the form

$$
f(t)=\left(\frac{2}{\pi}\right)^{1 / 2} \int_{0}^{1} \alpha_{1}(x) \cos (t x) d x, \quad g(t)=\left(\frac{2}{\pi}\right)^{1 / 2} \int_{0}^{1} \alpha_{2}(x) \sin (t x) d x,
$$

it is also easy to show that the system of coupled pairs of dual integral equations may be written in the form of the singular integral equation given below:

$$
p \phi(x)-\frac{d}{\pi} \int_{-1}^{1} \frac{\phi(t) d t}{t-x}=-\left(\frac{2}{\pi}\right)^{1 / 2}\left(h^{\prime}+s_{1}{ }^{\prime}\right), \quad|x|<1 .
$$

The integral equation (1.4) may be solved by standard solution techniques and the results are in agreement with those obtained by the method of reduction to the WienerHopf equation (1.2).

The problem solved by Spence for Bessel function kernels is also reduced to a singular integral equation by an alternate procedure. An appropriate selection of integral transforms in a form similar to (1.3) is found to reduce his coupled pairs of dual integral equations to the form of (1.4) with $p$ and $d$ appropriately defined.

2. The Wiener-Hopf equation. Formal differentiation of $(1.1 a)$ and (1.1c), which 
can be shown to be justified, leads at once to the following sine and consine transform equations:

$$
\begin{aligned}
F_{s}(\rho) & =-\frac{1}{a_{1}} h^{\prime}(\rho)-\frac{a_{2}}{a_{1}} G_{s}(\rho), & & \rho<1, \\
& =\frac{1}{b_{3}} s_{2}(\rho)-\frac{b_{4}}{b_{3}} G_{s}(\rho), & & \rho<1,
\end{aligned}
$$

and

$$
\begin{aligned}
F_{c}(\rho) & =\frac{1}{b_{1}} s_{1}{ }^{\prime}(\rho)-\frac{b_{2}}{b_{1}} G_{c}(\rho), & & \rho<1, \\
& =\frac{1}{a_{3}} h_{2}(\rho)-\frac{a_{4}}{a_{3}} G_{c}(\rho), & & \rho<1,
\end{aligned}
$$

where $F_{s}(\rho), F_{c}(\rho), G_{s}(\rho)$ and $G_{c}(\rho)$ denote the Fourier sine and cosine transforms of the function $f(t)$ and $g(t)$. Inversion of (2.1) and (2.2) followed by interchange of orders of integration (which can be proved to be justified) leads to Eq. (2.3) below for $t>0$. The only difficulty in this process arises from the integrals of the form $\int_{0}^{\infty} \cos u t d u$ $\int_{0}{ }^{\infty} g(x) \cos x u d x$ which can be reduced to the Fourier single-integral formula upon treatment of the integral as $\lim _{\lambda \rightarrow \infty} \int_{0}^{\lambda} \cos u t d u \int_{0}^{\infty} g(x) \cos x u d u$. Hence, if it is assumed that $g(x)$ is continuous and satisfies the condition $g \in L^{1}(-\infty, \infty)$ and that $g(x)$ is of bounded variation [12], the result below is immediate:

$$
\begin{aligned}
f(t)= & \frac{2}{\pi}\left\{\frac{1}{b_{3}} D_{2}(t)-\frac{1}{a_{1}} H_{1}(t)\right\} \\
& +\left(\frac{b_{4}}{b_{3}}-\frac{a_{2}}{a_{1}}\right) \int_{0}^{\infty} g(u)[k(u-t)-k(u+t)] d u-\frac{b_{4}}{b_{3}} g(t), \\
f(t)= & \frac{2}{\pi}\left\{\frac{1}{b_{1}} D_{1}(t)+\frac{1}{a_{3}} H_{2}(t)\right\} \\
& +\left(\frac{a_{4}}{a_{3}}-\frac{b_{2}}{b_{1}}\right) \int_{0}^{\infty} g(u)[k(u-t)+k(u+t)] d u-\frac{a_{4}}{a_{3}} g(t),
\end{aligned}
$$

where

$$
\begin{array}{ll}
H_{1}(t)=\int_{0}^{1} h_{1}{ }^{\prime}(\rho) \sin \rho t d \rho ; & H_{2}(t)=\int_{1}^{\infty} h_{2}(\rho) \cos \rho t d \rho, \\
D_{1}(t)=\int_{0}^{1} s_{1}{ }^{\prime}(\rho) \cos \rho t d \rho ; & D_{2}(t)=\int_{1}^{\infty} s_{2}(\rho) \sin \rho t d \rho .
\end{array}
$$

Eliminating $f(t)$ from Eqs. (2.3), we obtain

$$
g(t)=\frac{1}{\omega} c(t)+\frac{\beta_{1}}{\omega} \int_{0}^{\infty} g(u) k(u+t) d u+\frac{\beta_{2}}{\omega} \int_{0}^{\infty} g(u) k(u-t) d u \quad t>0
$$

where

$$
\begin{aligned}
\omega & =\left(a_{4} / a_{3}\right)-\left(b_{4} / b_{3}\right), \quad \omega \neq 0, \\
c(t) & =\frac{2}{\pi}\left[\frac{1}{b_{1}} D_{1}(t)+\frac{1}{a_{3}} H_{2}(t)-\frac{1}{b_{3}} D_{2}(t)+\frac{1}{a_{1}} H_{1}(t)\right],
\end{aligned}
$$


and

$$
\beta_{1}=\frac{a_{4}}{a_{3}}-\frac{b_{2}}{b_{1}}+\frac{b_{4}}{b_{3}}-\frac{a_{2}}{a_{1}}, \quad \beta_{2}=\frac{a_{4}}{a_{3}}-\frac{b_{2}}{b_{1}}-\frac{b_{4}}{b_{3}}+\frac{a_{2}}{a_{1}} .
$$

An equation of the type (2.5) has been solved by Spence [2] for the case $\beta_{1}=0, \beta_{2}$ and $\omega$ real coefficients. In the next section it will be shown how the system (1.1) can be transformed so that $\beta_{1}=0$.

3. The equivalent system. Define two functions $\psi_{1}(t)$ and $\psi_{2}(t)$ as follows:

$$
\psi_{1}(t)=f(t), \quad \psi_{2}(t)=g(t)+\delta f(t)
$$

where $\delta$ is a parameter to be determined. By means of (3.1) system (1.1) is transformed into the equivalent system

$$
\begin{aligned}
& \int_{0}^{\infty} t^{-1}\left[A_{1} \psi_{1}(t)+A_{2} \psi_{2}(t)\right] \cos \rho t d t=h_{1}(\rho) \\
& \int_{0}^{\infty}\left[A_{3} \psi_{1}(t)+A_{4} \psi_{2}(t)\right] \cos \rho t d t=h_{2}(\rho) \\
& \int_{0}^{\infty} t^{-1}\left[B_{1} \psi_{1}(t)+B_{2} \psi_{2}(t)\right] \sin \rho t d t=s_{1}(\rho) \\
& \int_{0}^{\infty}\left[B_{3} \psi_{1}(t)+B_{4} \psi_{2}(t)\right] \sin t d t=s_{2}(\rho)
\end{aligned}
$$

where

$$
\begin{array}{llll}
A_{1}=a_{1}-\delta a_{2}, & A_{2}=a_{2}, & A_{3}=a_{3}-\delta a_{4}, & A_{4}=a_{4}, \\
B_{1}=b_{1}-\delta b_{2}, & B_{2}=b_{2}, & B_{3}=b_{3}-\delta b_{4}, & B_{4}=b_{4} .
\end{array}
$$

In view of its similarity to the system (1.1), the system (3.2) would obviously reduce to a Wiener-Hopf integral equation

$$
\psi_{2}(t)=\frac{1}{\omega^{\prime}} C(t)+\frac{\beta_{1}{ }^{\prime}}{\omega^{\prime}} \int_{0}^{\infty} \psi_{2}(u) k(u+t) d u+\frac{\beta_{2}{ }^{\prime}}{\omega^{\prime}} \int_{0}^{\infty} \psi_{2}(u) k(u-t) d u, \quad t>0
$$

similar to (2.5), and to

$$
\begin{aligned}
\int_{0}^{\infty} & \psi_{2}(u)[k(\mu-t)+k(u+t)] d u \\
& =\frac{1}{\left(\frac{A_{4}}{A_{3}}-\frac{B_{2}}{B_{1}}\right)}\left\{\frac{A_{4}}{A_{3}} \psi_{2}(t)+\psi_{1}(t)-\frac{2}{\pi}\left[\frac{1}{B_{1}} D_{1}(t)+\frac{1}{A_{3}} H_{2}(t)\right]\right\}, \quad t>0,
\end{aligned}
$$

similar to (2.3), where

$$
\begin{aligned}
\beta_{1}{ }^{\prime} & =\frac{B_{4}}{B_{3}}-\frac{B_{2}}{B_{1}}+\frac{A_{4}}{A_{3}}-\frac{A_{2}}{A_{1}}, \quad \beta_{2}{ }^{\prime}=\frac{A_{4}}{A_{3}}-\frac{B_{2}}{B_{1}}-\frac{B_{4}}{B_{3}}+\frac{A_{z}}{A_{1}}, \\
\omega^{\prime} & =\frac{A_{4}}{A_{3}}-\frac{B_{4}}{B_{3}}, \quad \omega^{\prime} \neq 0, \quad \beta=-\beta_{2}{ }^{\prime} / \omega^{\prime},
\end{aligned}
$$




$$
C(t)=\frac{2}{\pi}\left[\frac{1}{B_{1}} D_{1}(t)+\frac{1}{A_{3}} H_{2}(t)-\frac{1}{B_{3}} D_{2}(t)+\frac{1}{A_{1}} H_{1}(t)\right] .
$$

It can be shown that $\beta_{1}{ }^{\prime}=0$ if $\delta$ satisfics the quadratic

$$
A^{\prime} \delta^{2}+2 B^{\prime} \delta+C^{\prime}=0
$$

where

$$
\begin{aligned}
& A^{\prime}=a_{2} a_{4} b_{1} b_{4}-a_{2} a_{3} b_{4} b_{2}-a_{2} a_{4} b_{2} b_{3}+a_{1} a_{4} b_{2} b_{4}, \\
& B^{\prime}=a_{2} a_{3} b_{2} b_{3}-a_{1} a_{4} b_{1} b_{4}, \\
& C^{\prime}=a_{1} a_{3} b_{1} b_{4}-a_{1} a_{3} b_{2} b_{3}-a_{2} a_{3} b_{1} b_{3}+a_{1} a_{4} b_{1} b_{3} .
\end{aligned}
$$

The parameter $\delta$ is generally complex. Hence the coefficients $A_{i}, B_{i}(i=1-4)$ as well as $\beta_{2}{ }^{\prime}$ and $\omega^{\prime}$ become complex.

4. Solution. There always exists a solution to (3.8); therefore the transformation (3.1) always succeeds in producing an equivalent system for which $\beta_{1}{ }^{\prime}=0$. In [1] Spence's results for the Wiener-Hopf equation with complex coefficients were quoted; we use those results in this paper. Let $\psi_{2}(t)=0$ for $t<0$; then (3.4) can be extended over $-\infty<x<\infty$ as follows:

$$
e(x)=\psi_{2}(x)+\beta \int_{-\infty}^{\infty} \psi_{2}(u) k(u-x) d u-\frac{1}{\omega} C(t),
$$

where $e(x)$ is an unknown function which must be zero for $x>0$. Replacing $x$ by $-x$ in (4.1) and adding the resulting equation to (4.1), one finds for $x>0$

$e(-x)=\psi_{2}(x)+\beta \int_{-\infty}^{\infty} \psi_{2}(u)[k(u+x)+k(u-x)] d u-\frac{4}{\omega^{\prime} \pi}\left\{\frac{1}{B_{1}} D_{1}(x)+\frac{1}{A_{3}} H_{2}(x)\right\}$.

Now, upon substitution from (3.5), one gets

$\psi_{1}(x)=-\frac{\gamma}{\beta}\left\{\left[1+\frac{A_{4}}{A_{3}} \frac{\beta}{\gamma}\right] \psi_{2}(x)-e(-x)\right\}+\frac{2}{\pi}\left(1+\frac{2}{\omega^{\prime}} \frac{\gamma}{\beta}\right)\left[\frac{1}{B_{1}} D_{1}(x)+\frac{1}{A_{3}} H_{2}(x)\right]$

where

$$
\gamma=\left(A_{4} / A_{3}\right)-\left(B_{2} / B_{1}\right) .
$$

The solution of the system (3.2) consists in solving (4.1) for $\psi_{2}(x)$ and $e(x)$ and using (4.3) to find $\psi_{1}(x)$. The functions $f(t)$ and $g(t)$ can then be determined from (3.1).

Let $C(t)$ be of the form

$$
C(t)=P\left(\frac{d}{d t}\right) k(t)+Q\left(\frac{d}{d t}\right) l(t)
$$

where $P$ and $Q$ are $N$ th degree polynomials in the operator $d / d t$, and $l(t)=(1-\cos t) / \pi t$. The function $C(t)$ is of the form (4.5), for example, when $h_{1}(\rho)$ and $s_{1}(\rho)$ are polynomials and $h_{2}(\rho)=s_{2}(\rho)=0$. It can be shown [1] that the $L^{\prime}(-\infty, \infty)$ solution of (4.1) is

$$
\begin{gathered}
\psi_{2}(t) \\
e(-t)
\end{gathered}= \pm \frac{2}{\pi} \sinh \frac{1}{2} \pi \kappa \int_{0}^{1}[U(w) \cos \phi+w V(w) \sin \phi] d w
$$


where $\phi=k \theta(w) \pm w x$ (the \pm sign corresponds respectively to $\psi_{2}(t)$ and $e(-t)$ ), $\kappa$ is the known complex parameter

$$
\kappa=\frac{1}{\pi} \log (1+\beta), \quad \theta(w)=\frac{1}{2} \log \frac{1+\frac{w}{1}}{w}, \quad|w|<1
$$

and

$$
\begin{aligned}
U(w)= & -\frac{\lambda}{2 \beta}\left(\frac{2}{\pi} \sinh \frac{1}{2} \pi \kappa\right)\left\{B_{1}(w) \psi(w, \kappa)+i w B_{2}(w) \chi(w, \kappa)\right\} \\
& -\frac{\lambda}{2 \beta}[S(w)-S(-w)]+\frac{\lambda}{2 \beta}[R(w)+R(-w)], \\
U(w)= & -\frac{\lambda i}{2 \beta w}\left(\frac{2}{\pi} \sinh \frac{1}{2} \pi \kappa\right)\left\{B_{2}(w) \psi(w, \kappa)+i w B_{1}(w) \chi(w, \kappa)\right\} \\
& +\frac{\lambda i}{2 \beta w}[S(w)-S(-w)]-\frac{\lambda i}{2 \beta w}[R(w)-R(-w)] .
\end{aligned}
$$

The parameter $\lambda$ used above is defined by $\lambda=1 / \omega^{\prime}$. The definitions of other symbols in (4.8) and (4.9) are as follows:

$$
\left.\begin{array}{l}
\psi(w, \kappa) \\
\chi(w, \kappa)
\end{array}\right\}=\operatorname{cosech} \frac{1}{2} \pi \kappa \int_{0}^{1 / 2}\left\{\begin{array}{c}
\sinh \kappa y \cot y \\
\cosh \kappa y
\end{array}\right\} \frac{d y}{\cos ^{2} y+w^{2} \sin ^{2} y}
$$

and

$$
\left.\begin{array}{l}
B_{1}(w) \\
B_{2}(w)
\end{array}\right\}=Q(i w) \pm Q(-i w) .
$$

The functions $R(w)$ and $S(w)$ in (4.8) and (4.9) are polynomials. The method of constructing them from $P(-i w)$ and $Q(-i w)$ is described by Spence. In the case of the second-degree polynomials

$$
P\left(\frac{d}{d t}\right)=p_{0}+p_{1} \frac{d}{d t}+p_{2}\left(\frac{d}{d t}\right)^{2} ; \quad Q\left(\frac{d}{d t}\right)=q_{1} \frac{d}{d t}+q_{2}\left(\frac{d}{d t}\right)^{2},
$$

$R(w)$ and $S(w)$ are found to be

$$
\begin{aligned}
& R(w)=p_{0}+\kappa p_{1}+\frac{1}{2} \kappa^{2} p_{2}-p_{2} w^{2}-i w\left(p_{1}+\kappa p_{2}\right), \\
& S(w)=\frac{2}{\pi} \sinh \frac{1}{2} \pi \kappa\left(q_{1} I_{0}+q_{2} I_{1}-i w q_{2} I_{0}\right),
\end{aligned}
$$

where

$$
I_{0}=\kappa \psi(1, \kappa), \quad I_{1}=\frac{1}{2}-\frac{1}{2} \kappa I_{0} .
$$

5. Punch in steady motion. Let a punch be in steady motion on an elastic halfspace $y \leq 0,-\infty<x<\infty$, where the $x$-coordinate is relative to the center of the region of contact which moves with a velocity $c$. It is assumed that the normal contact stress is always compressive and that there is friction between the punch and the halfspace on $y=0$. The boundary conditions are: 


$$
\begin{aligned}
\sigma_{y} & =\tau_{x y}=0, & & |x|>b, \\
\tau_{x y} & =-f \sigma_{y}, & & |x|<b, \\
v & =l(x), & & |x|<b,
\end{aligned}
$$

where $\sigma_{x}, \sigma_{y}, \tau_{x y}, u, v$ are stresses and displacements appropriate to a state of plane strain and $f$ is a coefficient of kinetic friction. It is easily shown that

$$
u=\frac{\partial \phi}{\partial x}+\frac{\partial \psi}{\partial y}, \quad v=\frac{\partial \phi}{\partial y}-\frac{\partial \psi}{\partial x}
$$

will satisfy the equations of equilibrium if

$\phi(x, y)=\int_{-\infty}^{\infty} A(\xi) \exp \left(|\xi| \alpha_{1} y-i \xi x\right) d \xi, \quad \psi(x, y)=\int_{-\infty}^{\infty} B(\xi) \exp \left(|\xi| \alpha_{2} y-i \xi x\right) d \xi$

where $\alpha_{i}{ }^{2}=1-c^{2} / c_{i}{ }^{2}(i=1,2)$. Here ${c_{1}}^{2}=(\lambda+2 \mu) / \rho, c_{2}{ }^{2}=\mu / \rho$, where $\lambda, \mu$ are Lamé constants. By writing

$(2 \pi)^{1 / 2} \xi^{3} \Delta A=\left(1+\alpha_{2}^{2}\right) \xi C-2 i \alpha_{2}|\xi| D,(2 \pi)^{1 / 2} \xi^{3} \Delta B=2 i \alpha_{1}|\xi| C-\left(1+\alpha_{2}^{2}\right) \xi D$,

where $\Delta=\left(1+\alpha_{2}\right)^{2}-4 \alpha_{1} \alpha_{2}$, and using the boundary condition (5.2), one obtains $D=-f C$. The function $C(\xi)$ may be written in the form $C=E+i F$ where $E$ and $F$ are even and odd functions respectively. The above relations allows the displacements and stresses to be written as sine and cosine transforms thus:

$$
\begin{aligned}
v_{c} & =-\left(\frac{2}{\pi}\right)^{1 / 2} \Delta^{-1} \int_{0}^{\infty} \xi^{-1}\left\{\alpha_{1}\left(1-\alpha_{2}{ }^{2}\right) E+f\left[2 \alpha_{1} \alpha_{2}-\left(1+\alpha_{2}{ }^{2}\right)\right] F\right\} \cos \xi x d \xi, \\
v_{s} & =\left(\frac{2}{\pi}\right)^{1 / 2} \Delta^{-1} \int_{0}^{\infty} \xi^{-1}\left\{-f\left[2 \alpha_{1} \alpha_{2}-\left(1+\alpha_{2}{ }^{2}\right)\right] E+\alpha_{1}\left(1-\alpha_{2}{ }^{2}\right) F\right\} \sin \xi x d \xi, \\
\sigma_{\nu c} / \mu & =\left(\frac{2}{\pi}\right)^{1 / 2} \int_{0}^{\infty} E \cos \xi x d \xi, \\
\sigma_{y s} / \mu & =\left(\frac{2}{\pi}\right)^{1 / 2} \int_{0}^{\infty} F \sin \xi x d \xi,
\end{aligned}
$$

where in (5.7)-(5.10) it is assumed that the boundary conditions can be written as even and odd functions. If this is not the case, then the same results can be developed directly from the exponential transforms as in Brilla [6].

Two types of indentors are considered, a cylindrical and a wedge punch; thus the boundary conditions will enter into (5.3) as

$$
\begin{array}{ll}
l(x)=-v_{0}+(\delta-x)^{2} / 2 R & \text { (cylindrical punch), } \\
l(x)=-a+\alpha x & \text { (wedge punch). }
\end{array}
$$

In the next section we will look into these specific examples.

6. Cylindrical punch. Use of the boundary conditions (5.1)-(5.3) and Eqs. (5.7)(5.10), with $l(x)$ defined by (5.11), leads to the following system of coupled pairs of dual integral equations appropriate for the cylinder: 


$$
\begin{array}{ll}
\int_{0}^{\infty} \xi^{-1}[d E(\xi)+p F(\xi)] \cos \xi x d \xi=h_{1}(x), & x<1, \\
\int_{0}^{\infty} E(\xi) \cos \xi x d \xi=0, & x>1, \\
\int_{0}^{\infty} \xi^{-1}[-p E(\xi)+d F(\xi)] \sin \xi x d \xi=s_{1}(x), & x<1, \\
\int_{0}^{\infty} F(\xi) \sin \xi x d \xi=0, & x>1,
\end{array}
$$

where the change of variable has been made so that the point $x=1$ corresponds to $x=b$ in the physical situation. The coefficients $d$ and $p$ and the functions $h_{1}(x)$ and $s_{1}(x)$ are defined below:

$$
\begin{gathered}
d=\alpha_{1}\left(1-\alpha_{2}{ }^{2}\right), \quad p=f\left[2 \alpha_{1} \alpha_{2}-\left(1+\alpha_{2}{ }^{2}\right)\right] \\
h_{1}(x)=\frac{\Delta}{b}\left(\frac{\pi}{2}\right)^{1 / 2}\left[v_{0}-\frac{\delta^{2}}{2 R}-\frac{x^{2} b^{2}}{2 R}\right], \quad s_{1}(x)=\frac{\Delta}{b}\left(\frac{\pi}{2}\right)^{1 / 2}\left(\frac{x b \delta_{0}}{R}\right) .
\end{gathered}
$$

Comparing Eqs. (6.1) and (6.2) with the system (1.1) shows that in the former case the coefficient $b_{3}=0$, which was not allowed. However, the following transformation removes that difficulty. Let

$$
E(\xi)=E(\xi), \quad F(\xi)=E(\xi)-P(\xi) ;
$$

then (6.1) and (6.2) become

$$
\begin{array}{ll}
\int_{0}^{\infty} \xi^{-1}[(d+p) E(\xi)-p P(\xi)] \cos \xi x d \xi=h_{1}(x), & x<1, \\
\int_{0}^{\infty} E(\xi) \cos \xi x d \xi=0, & x>1, \\
\int_{0}^{\infty} \xi^{-1}[(d-p) E(\xi)-d P(\xi)] \sin \xi x d \xi=s_{1}(x), & x<1, \\
\int_{0}^{\infty}[E(\xi)-P(\xi)] \sin x \xi d \xi=0, & x>1,
\end{array}
$$

where, in comparing these equations with (1.1) and (1.2), one finds

$$
\begin{array}{llll}
a_{1}=d+p, & a_{2}=-p, & a_{3}=1, & a_{4}=0, \\
b_{1}=d-p, & b_{2}=-d, & b_{3}=1, & b_{4}=-1,
\end{array}
$$

The above coefficients give $\delta=-(1 \pm i)$ as the solution of (3.8). Either value of $\delta$ may be used to obtain the solution. Using $\delta=-(1+i)$, one finds (from 3.3)

$$
\begin{aligned}
& A_{1}=d-i p, \quad A_{2}=-p, \quad A_{3}=1, \quad A_{4}=0, \\
& B_{1}=-i(d-i p), \quad B_{2}=-d, \quad B_{3}=-i, \quad B_{4}=-1 .
\end{aligned}
$$

Eqs. (3.6), (3.7) and (4.4) give

$$
\beta=-\frac{2 d}{d-i p}, \quad \omega^{\prime}=1, \quad \gamma=-\frac{d}{i(d-i p)}
$$


and (4.3) gives

$$
\psi_{1}(x)=\frac{i}{2}\left[\psi_{2}(x)-e(-x)\right], \quad x>0,
$$

where the functions $\psi_{1}$ and $\psi_{2}$ are related to $E(\xi)$ and $P(\xi)$ above according to the following relationship:

$$
E(\xi)=\psi_{1}(\xi), \quad P(\xi)=\psi_{2}(\xi)+\delta \psi_{1}(\xi) .
$$

The functions $\psi_{2}(\xi)$ and $e(-\xi)$ are defined by Eq. (4.6), where

$$
U(w)=\frac{\Delta}{R d}\left(\frac{\pi}{2}\right)^{1 / 2}\left[-\delta_{0}+\kappa b i\right], \quad V(w)=-\frac{\Delta b}{R d}\left(\frac{\pi}{2}\right)^{1 / 2},
$$

where

$$
\kappa=\frac{1}{\pi} \log (1+\beta)=\frac{1}{\pi} \log \left(-\frac{d+i p}{d-i p}\right)
$$

which can be written in the form

$$
\kappa=-(1+2 \alpha+2 m) i, \quad d \pm i p=\frac{d}{\cos \pi \alpha} \exp ( \pm i \pi \alpha) .
$$

The integer $m$ takes values $0, \pm 1, \pm 2, \cdots$. On using Eqs. (6.10)-(6.14), (6.4) and (4.6) we find

$$
\begin{aligned}
E(\xi)= & \left(\frac{2}{\pi}\right)^{1 / 2} \frac{\Delta}{R d} \sinh \frac{1}{2} \pi \kappa \\
& \cdot\left\{i\left(-\delta_{0}+i_{\kappa} b\right) \int_{0}^{1} \cos \kappa \theta \cos w \xi d w+b \int_{5}^{1} w \sin \kappa \theta \cos w \xi d w\right\}, \\
F(\xi)= & \left(\frac{2}{\pi}\right)^{1 / 2} \frac{\Delta}{R d} \sinh \frac{1}{2} \pi_{\kappa} \\
& \cdot\left\{\left(-\delta_{0}+i_{\kappa} b\right) \int_{0}^{1} \sin \kappa \theta \sin w \xi d w+b i \int_{0}^{1} w \cos \kappa \theta \sin w \xi d w\right\},
\end{aligned}
$$

which completes the solution of the coupled pairs of dual integral equations for the cylinder.

The normal stress. The normal stress can be computed from the formula

$$
\sigma_{y} / \mu=(2 \pi)^{-1 / 2} \int_{-\infty}^{\infty}(E+i F) \exp (-i \xi x) d \xi
$$

which can be written in the form

$$
\sigma_{\nu} / \mu=\left(\frac{2}{\pi}\right)^{1 / 2} \frac{d}{d x}\left\{\int_{0}^{\infty} \xi^{-1} E(\xi) \sin \xi x d \xi-\int_{0}^{\infty} \xi^{-1} F(\xi) \cos \xi x d \xi\right\},
$$

Substitution of $E(\xi)$ and $F(\xi)$ from (6.15) and (6.16) into (6.18) gives

$$
\sigma_{\nu} / \mu=\frac{\Delta i}{R d} \sinh \frac{1}{2} \pi \kappa\left\{\left(-\delta_{0}+i_{\kappa} b\right)+b \xi\right\} \exp \left[-i_{\kappa} \theta(\xi)\right] .
$$


Since $\kappa=-(1+2 \alpha+2 m) i$, we find that $\sinh \frac{1}{2} \pi \kappa=-i \cos (\alpha+m) \pi$ and

$$
\begin{aligned}
\sigma_{\nu} / \mu= & \frac{\Delta}{R d} \cos \pi(\alpha+m) \\
& \cdot\left\{\left[-\delta_{0}+2 \alpha b+2 m b\right]\left(\frac{1+\xi}{1-\xi}\right)^{-1 / 2-\alpha-m}+b(1+\xi)^{1 / 2-\alpha-m}(1-\xi)^{1 / 2+\alpha+m}\right\} .
\end{aligned}
$$

From the physical standpoint it is known that the normal stress must not be singular at $\xi= \pm 1$. Hence, since $0<\alpha<\frac{1}{2}$,

$$
\delta_{0}=+2 \alpha b+2 m b, \quad-\frac{1}{2}<m<\frac{1}{2} .
$$

Being an integer, therefore, $m$ can be only zero, leading at once to the result (after the transformation $\xi=x / b$ )

$$
\sigma_{\nu} / \mu=\frac{\Delta}{R d} \cos \pi \alpha(b+x)^{1 / 2-\alpha}(b-x)^{1 / 2+\alpha}, \quad-b \leq x \leq b .
$$

7. Wedge punch. For a wedge punch the same coupled pair of dual integral equations is used with the boundary conditions

$$
h_{1}(x)=0, \quad S_{1}(x)=\frac{\Delta}{b}\left(\frac{\pi}{2}\right)^{1 / 2} x b \epsilon,
$$

which leads to

$$
C(x)=p_{0} k(x), \quad p_{0}=\frac{-2 \Delta\left(\frac{\pi}{2}\right)^{1 / 2} \epsilon}{d-i p} i .
$$

The functions $U(w)$ and $V(w)$ become

$$
U(w)=\frac{\Delta}{d}\left(\frac{\pi}{2}\right)^{1 / 2} e, \quad V(w)=0,
$$

resulting in the following solution of the system of dual integral equations:

$$
\begin{aligned}
& E(x)=\frac{\Delta}{d}\left(\frac{2}{\pi}\right)^{1 / 2} \epsilon i \sinh \frac{1}{2} \pi \kappa \int_{0}^{1} \cos \kappa \theta \cos w x d w \\
& F(x)=\frac{\Delta}{d}\left(\frac{2}{\pi}\right)^{1 / 2} \epsilon \sinh \frac{1}{2} \pi \kappa \int_{0}^{1} \sin \kappa \theta \sin w x d w .
\end{aligned}
$$

Use of this solution and $\kappa=-(1+2 \alpha+2 m) i$ gives

$$
\frac{\sigma_{y}}{\mu}=\frac{\Delta \epsilon}{d} \cos \pi(\alpha+m)\left(\frac{1+\xi}{1-\xi}\right)^{-(1 / 2+\alpha+m)}
$$

where $m$ is an integer. Physical considerations permit that the normal stress be singular at $\xi=-1$ but not at $\xi=+1$, thus demanding that $m<\frac{1}{2}+\alpha$, or simply that $m<1$ (as $0<\alpha<\frac{1}{2}$ ). Moreover, the normal stress (7.5) must be integrable, which requires $m>-1$. Being an integer, therefore, $m$ can be only zero. Hence the normal stress becomes 


$$
\sigma_{y} / \mu=\frac{\Delta \epsilon}{d} \cos \pi \alpha(b+x)^{-1 / 2-\alpha}(b-x)^{1 / 2+\alpha}
$$

which agrees with Muskhelishvili [13] when $c=0$.

8. Singular integral equation approach. Now consider Eqs. (6.1) and (6.2) from the point of view of writing the system as a singular integral equation rather than a WienerHopf equation. It is convenient to write

$$
\begin{aligned}
& E(\xi)=\left(\frac{2}{\pi}\right)^{1 / 2} \int_{0}^{1} \tau_{1}(x) \cos \xi x d \xi, \\
& F(\xi)=\left(\frac{2}{\pi}\right)^{1 / 2} \int_{0}^{1} \tau_{2}(x) \sin \xi x d \xi,
\end{aligned}
$$

where $\tau_{1}$ and $\tau_{2}$ are even and odd, respectively, and (8.1) satisfies the second of (6.1) and (6.2) respectively. The following relations [14] are noted:

$$
\begin{aligned}
& \int_{0}^{\infty} \xi^{-1} \cos \xi s \cos \xi x d \xi=-\frac{1}{2} \log |(x+s)(x-s)|+c, \\
& \int_{0}^{\infty} \xi^{-1} \sin \xi s \sin \xi x d \xi=\frac{1}{2} \log |(x+s) /(x-s)|, \\
& \int_{0}^{\infty} \xi^{-1} \cos \xi s \sin \xi x d \xi=\frac{1}{2} \pi H(x-s), \\
& \int_{0}^{\infty} \xi^{-1} \sin \xi s \cos \xi x d \xi=\frac{1}{2} \pi H(s-x),
\end{aligned}
$$

where $c$ is an arbitrary constant. Substituting (5.5) and (5.6) into (5.1) and (5.3), differentiating, and making use of the symmetric properties of $\tau_{1}$ and $\tau_{2}$, we obtain the following equation:

$$
\begin{aligned}
\frac{d}{\pi} \int_{-1}^{1} \frac{\tau_{1}(s) d s}{s-x}-p \tau_{2}(x)=\left(\frac{2}{\pi}\right)^{1 / 2} h_{1}{ }^{\prime}(x), & |x|<1 . \\
-p \tau_{1}(x)+d \int_{-1}^{1} \frac{\tau_{2}(s) d s}{s-x}=\left(\frac{2}{\pi}\right)^{1 / 2} s_{1}{ }^{\prime}(x), & |x|<1 .
\end{aligned}
$$

Since $\sigma_{y}=\sigma_{y c}+\sigma_{y s}$, it is clear that,

$$
\phi=\sigma_{y} / \mu=\tau_{1}(x)+\tau_{2}(x)
$$

and the two equations in (8.3) are added to obtain

$$
p \phi(x)-\frac{d}{\pi} \int_{-1}^{1} \frac{\phi(t) d t}{t-x}=-\left(\frac{2}{\pi}\right)^{1 / 2}\left(h_{1}{ }^{\prime}+{s_{1}}^{\prime}\right), \quad|x|<1 .
$$

Now let

$$
\Phi(z)=\frac{1}{2 \pi i} \int_{-1}^{1} \frac{\phi(t) d t}{t-z},
$$

where according to the Plemelj relations 


$$
\phi(t)=\Phi^{+}-\Phi^{-}, \quad \frac{1}{\pi i} \int_{-1}^{1} \frac{\phi(t) d t}{t-z}=\Phi^{+}+\Phi^{-},
$$

and Eq. (8.5) is reduced to a Hilbert problem

$$
\Phi^{+}-g \Phi^{-}=\frac{(2 / w)^{1 / 2}}{i(d+i p)}\left(h_{1}{ }^{\prime}+s_{1}{ }^{\prime}\right)
$$

where

$$
g=-(d-i p) /(d+i p)=-\exp (2 i \pi \alpha)
$$

A. Cylindrical punch. For this case

$$
h_{1}^{\prime}(x)=-\left(\frac{\pi}{2}\right)^{1 / 2} \Delta x / R, \quad s_{1}{ }^{\prime}(x)=\left(\frac{\pi}{2}\right)^{1 / 2} \Delta \delta_{0} / R
$$

and it is easy to verify that a solution to (8.8) and (8.10) bounded at the end points is

$$
\phi(x)=\frac{\cos \pi \alpha}{d R} \Delta(b+x)^{1 / 2-\alpha}(b-x)^{1 / 2+\alpha} ; \quad-b \leq x \leq b
$$

where $\delta_{0}=2 \alpha b$ in agreement with (6.22).

B. Wedge punch. The boundary conditions for this case are

$$
h_{1}^{\prime}(x)=0, \quad s_{1}^{\prime}(x)=-\left(\frac{\pi}{2}\right)^{1 / 2} \Delta \epsilon
$$

and the solution to (8.8) and (8.13) bounded at the point $x=b$ is

$$
\phi(x)=\frac{\cos \pi \alpha}{d} \Delta \epsilon(b+x)^{-1 / 2-\alpha}(b-x)^{1 / 2+\alpha},
$$

in agreement with (7.6).

\section{REFERENCES}

[1] R. Khadem, On two pairs of simultaneous dual integral equations, J. Eng. Math. 5, 121-126 (1971)

[2] D. A. Spence, Wiener-Hopf equation arising in elastic contact problems, Proc. Roy. Soc. London A 305, 81-92 (1968)

[3] J. W. Craggs and A. M. Roberts, On the motion of a heavy cylinder over the surface on an elastic solid J. Appl. Mech. 34, 207-209 (1967)

[4] A. M. Roberts, A two-dimensional mixed boundary-values problem in elasticity, Quart. Appl. Math. 28, 445-449 (1970)

[5] A. M. Roberts, Further two-dimensional effects of cylinders rolling on an elastic half-space, Quart. Appl. Math. 29, 17-28 (1971)

[6] J. Brilla, Contact problems of an elastic anisotropic half-plane, Revue Mech. Appl. Buc. 7, 617-642 (1962)

[7] J. B. Alblas and M. Kuipers, Contact problems of a rectangular block on an elastic layer of finite thickness, Acta Mech. 9, 1-12 (1970)

[8] J. B. Alblas and M. Kuipers, On the two-dimensional problem of a cylindrical stamp pressed into a thin elastic layer, Acta Mech. 9, 292-311 (1970)

[9] J. B. Alblas and M. Kuipers, The two-dimensional conlact problem of a rough stamp sliding slowly on an elastic layer-general considerations and thick layer asymptotes, Int. J. Solids Structures 7, 99-109 (1971)

[10] J. B. Alblas and M. Kuipers, The Two-dimensional contact problem of a rough stamp stiding slowly on an elastic layer-II. Thin layer asymptotes, Int. J. Solids Structures 7, 225-237 (1971) 
[11] L. M. Keer and J. M. Freedman, Static response of a rigid strip bonded to an elastic layer, Acta Mechanica, 17, 1-15 (1973)

[12] E. C. Titchmarsh, Theory of Fourier integrals, Oxford at the Clarendon Press, 1962, p. 25

[13] N. I. Muskhelishvili, Some basic problems of the mathematical theory of elasticity, Noordhoff, Holland, p. 498

[14] M. J. Lighthill, Introduction to Fourier analysis and generalized functions, Cambridge University Press, 1959, p. 15

[15] D. A. Spence, Self-similar solutions tọ adhesive contact problems with incremental loading, Proc. Roy. Soc. London A 305, 5-80, (1968)

[16] L. M. Keer, Mixed boundary value-problems for an elastic half-space, Proc. Camb. Phil. Soc. 63, 1379-1386 (1967)

Appendix: Axially-symmetric case. In observing the relationship between the Wiener-Hopf and singular integral equation techniques for plane problems, one finds that similar results hold for the axially-symmetric case such as that treated by Spence. Indeed, if the indentation of a halfspace $z \geq 0$ is described by the coupled system

$$
\begin{array}{rlrl}
2 \mu u_{r}(r, 0) & =\int_{0}^{\infty}[-(1-2 \nu) D+2(1-\nu) E] J_{1}(\xi r) d \xi=2 \mu u, & r<1, \\
2 \mu u_{z}(r, 0)=\int_{0}^{\infty}[2(1-\nu) D-(1-2 \nu) E] J_{0}(\xi r) d \xi=2 \mu v, & r<1, \\
\tau_{z z}(r, 0)=-\int_{0}^{\infty} \xi D J_{0}(\xi r) d \xi=0, & r>1, \\
\tau_{z r}(r, 0)=-\int_{0}^{\infty} \xi E J_{1}(\xi r) d \xi=0, & r>1,
\end{array}
$$

it can be easily shown that the representation

$$
D=\int_{0}^{1} \phi(t) \cos (\xi t) d t, \quad E=\int_{0}^{1} \psi(t) \sin (\xi t) d t
$$

will satisfy (3) and (4) identically. Eqs. (1) and (2) can be transformed to the following:

$$
\begin{aligned}
& \frac{d}{d x} \int_{0}^{\infty}[-(1-2 \nu) D+2(1-\nu) E] \frac{\cos \xi x}{\xi} d \xi=-2 \mu \int_{0}^{x} \frac{(u r)^{\prime}}{\left(x^{2}-r^{2}\right)^{1 / 2}} d r-p(x), \\
& \int_{0}^{\infty}[2(1-\nu) D-(1-2 \nu) E] \frac{\sin \xi x}{\xi} d \xi=2 \mu \int_{0}^{x} \frac{r v}{\left(x^{2}-r^{2}\right)^{1 / 2}} d r=q(x) .
\end{aligned}
$$

Use of (5) and the relations in (8.2) gives, provided that $\phi(t)=\phi(-t)$ and $\psi(t)=\psi(-t)$,

$$
2(1-\nu) \Phi(x)+\frac{(1-2 v)}{\pi i} \int_{-1}^{1} \frac{\Phi(t) d t}{t-x}=\frac{2}{\pi}\left(q^{\prime}-i p\right), \quad-1 \leq x \leq 1,
$$

where

$$
\Phi=\phi-i \psi
$$

Eq. (8) can be solved easily by the techniques in [13] for polynomial indenters.

When $u=0, v=\epsilon$, then $p=0$ and $q^{\prime}=2 \mu \epsilon$. Let [13]

$$
\Phi=\chi^{+}-\chi^{-}, \quad \frac{1}{\pi i} \int_{-1}^{1} \frac{\Phi(t) d t}{t-x}=\chi^{+}+\chi^{-}
$$


Then

$$
(3-4 \nu) \chi^{+}-\chi^{-}=\frac{4}{\pi} \mu \epsilon,
$$

for which the solution is

$$
\chi(t)=\frac{2}{\pi} \frac{\mu \epsilon}{(1-2 \nu)}\{1-\mathrm{X}(t)\}
$$

where

$$
\mathbf{X}(t)=(t+1)^{-i \beta}(t-1)^{i \beta}, \quad \beta=\frac{\log (3-4 \nu)}{2 \pi} .
$$

Then, using (10), we get

$$
\Phi(t)=\frac{4}{\pi} \mu \in \mathrm{X}^{+}(t)
$$

Since

$$
P=2 \pi \int_{0}^{1} \tau_{z z} r d r=-\pi \operatorname{Re}\left\{\int_{-1}^{1} \Phi(t) d t\right\},
$$

the load is obtained as

$$
-P=\frac{4 \mu \epsilon}{1-2 \nu} \log (3-4 \nu),
$$

in agreement with Spence [15]. The approach used here is somewhat analogous to that given in [16], although one arrives at the pertinent results in a more straightforward manner by the present method. 